\title{
Antimicrobial Resistance Phenomenon
}

\author{
Moustafa El Shenawy* \\ Department of Food \& Environmental Microbiology, National Research Center, Egypt
}

*Corresponding author: Moustafa El Shenawy, Department of Food \& Environmental Microbiology, National Research Center, Egypt.

To Cite This Article: Moustafa El Shenawy. Antimicrobial Resistance Phenomenon. Am J Biomed Sci \& Res. 2019 - 2(4). AJBSR.MS.ID.000597. DOI: $10.34297 / A J B S R .2019 .02 .000597$

Received: April 09, 2019 | Published: April 23, 2019

\begin{abstract}
Antimicrobial resistance (AMR) is one of the most serious global public health threats in this century. The first World Health Organization (WHO) Global report on surveillance of AMR, published in April 2014, collected for the first-time data from national and international surveillance networks, showing the extent of this phenomenon in many parts of the world and also the presence of large gaps in the existing surveillance. This mini-review focus on the story of antibiotics, antibacterial resistance (ABR) phenomenon, how does antibiotics resistance occur and spread and, what can be done to go over this problem.
\end{abstract}

Keywords: Antibiotic Resistance; Multidrug Resistance Bacteria

\section{Introduction}

One of the most alarming consequences of antibiotic overuse is the emergence and spread of antibiotic resistant bacteria, which could bring «the end of modern medicine as we know it» (Margaret Chan, Director General of the World Health Organization, 2012) [1]. Antibiotic resistance occurs when bacteria lose their sensitivity to antibiotics. It develops when a bacterium mutates or acquires a resistance gene. Resistant bacteria are able to withstand attack by antibiotics, so that standard treatments become ineffective and infections persist and may spread to other people. Resistance of microbial pathogens to antibiotics is increasing worldwide at an accelerating rate, with a concomitant increase in morbidity and mortality associated with infections caused by antibiotic resistant pathogens. Thus, The Center for Disease Control and Prevention considers this phenomenon «one of the worlds`s most pressing health problems in the 21st century» [2]. This short review describes/gives short sight on the story of antibiotics, the resistant bacteria problem, how does antibiotics resistance occur, how can antibiotics resistance spread, as well as, what can be done to go over this dilemma.

\section{Discussion}

\section{The story of antibiotics}

On the morning of September 3rd, 1928, the discovery of the antibiotics began, accidently, by Professor Alexander Fleming when he was sorting through a number of glass plates which had previously been coated with Staphylococcus spp. bacteria as part of his research. Ten years later, Howard Florey and Ernst Chain, working at Oxford University, isolated the bacteria-killing substance found in the mold - penicillin. In 1945 Fleming and his coworkers were awarded the Nobel Prize for Medicine. Post-1945 the era of the antibiotics started to appear and had saved many lives. Since that time, and due to the diagnostic gap problem [which is the heart of resistant phenomena], the challenge to accurately distinguish between bacterial infections (which warrant antibiotic therapy) and viral infections (for which antibiotic treatment is generally not required) followed by wrong, under and / or over use of the antibiotics significantly increased the prevalence of antibiotic resistance bacteria all over the world. Nowadays, The Center for Disease Control and Prevention considers this phenomenon «one of the worlds`s most pressing health problems in the 21st century». Most recent, WHO announced in the World Antibiotic Awareness Week, 13-19 November 2017, that our time with antibiotics is running out and they are in danger of losing their effectiveness within 10 years, putting the world at risk of entering a "post-antibiotic era", in which antibiotics will no longer be effective against infectious diseases.

\section{Antibiotic resistance occurs}

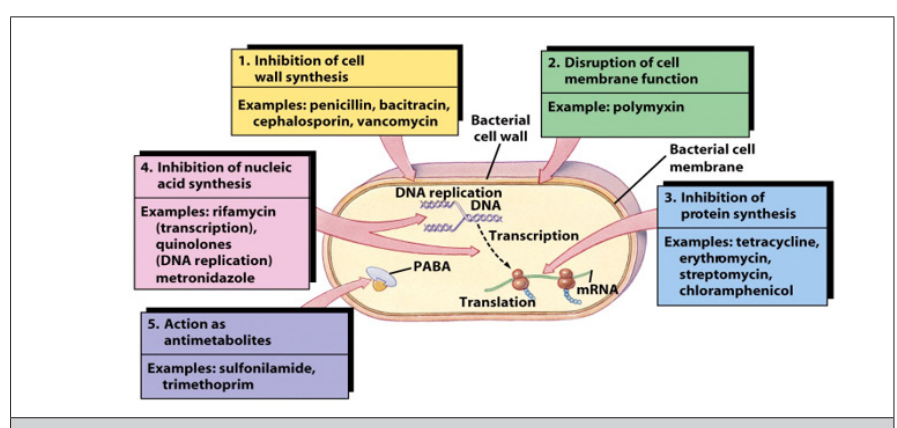

Figure 1: Microbiology, 6/e. 
when bacteria lose their sensitivity to antibiotics. It develops when a bacterium mutates or acquires a resistance gene. The mechanism can be happened in the bacterial cell as shown in the following (Figure 1).

\section{How can antibiotics resistance spread?}

With the growth of global trade and travel, resistance of microbial pathogens to antibiotics is increasing worldwide at an accelerating rate, in any part of the world leading to increase in morbidity and mortality. At least 2 million people are infected with antibiotic resistant bacteria each year in the US alone, and at least 23,000 people die as a direct result of these infections. In the European Union, an estimated 400,000 patients present with resistant bacterial strains each year, of which 25,000 patients die. There are three main interconnections make it easy for antibiotic resistant bacteria to jump from one to another. They are Human (people), Animals including birds and The environment.

\section{Factors Contributing to the Emergence \& Spread of} AMR

\section{Un-optimized use of antibiotics (antibiotic misuse)}

Un-optimized use of antibiotics is a significant public health concern and economic burden worldwide. Misuse relates both to overuse disease, such as a viral infection for which antibiotics are ineffective, and when treating a non-bacterial to underuse in cases of bacterial disease for which antibiotic treatment was delayed or not given and would have benefited the patient.

Antibiotic overuse is a common phenomenon, with the global overuse rate estimated at $40-70 \%$. For example, according to the US Centers for Disease Control and Prevention (CDC), over 60 million antibiotic prescriptions are given in the US annually to treat the flu, for which they are ineffective and inappropriate. Over-prescription of unnecessary antibiotics may cause adverse events such as allergic reactions, antibiotic-associated diarrhea, intestinal yeast infection, etc. These preventable adverse events negatively impact patient care and typically are associated with prolonged hospital stay. Antibiotic overuse is also driving the emergence of multi-drug resistant bacteria-one of the biggest healthcare problems of our time.

Antibiotic underuse in cases of bacterial disease for which antibiotic treatment was delayed or not given and would have benefited the patient. may lead to prolonged disease duration and increased rate of disease-related complications.

Counter antibiotics without prescription in pharmacies is another issue that is driving the improper use of antibiotics.

The use of antibiotics as a growth promoter in livestock and fish farming is an accepted practice in many industrialized countries.

Releasing large quantities of antibiotics into the environment during pharmaceutical manufacturing, and or the expired drugs in hospital through inadequate wastewater treatment.

\section{Limitation of current diagnostic tools}

This leads to the Extended time to Laboratories results. It requires hours to days to provide information, whereas doctors need to prescribe the antibiotic within min.(s) during the patient visit. Failure infection diagnosis when site is inaccessible. Usually direct sampling is used, however this is not easy if the infection site is inaccessible such as in the case middle-ear infection and bronchitis. False-results due to carriage of natural flora. Many bacteria live in our bodies as natural flora. For example, S. pneumonia exist in the upper respiratory tract, as natural flora, of $70 \%$ of healthy children and $25 \%$ of adults. Thus, the diagnostic can lead to false alarm/ results and consequently over prescription of antibiotics.

\section{Others}

Poor personal hygiene and sanitation such as regularly washing hands. Poor infection control in health care setting like Poor Hygiene Practices in Hospitals .Patients not finishing the entire antibiotic course. Absence of new antibiotics being discovered. It is uncertain whether antibacterials in soaps and other products contribute to antibiotic resistance, but antibacterial soaps are discouraged for other reasons.

\section{What is currently being done to go over this dilemma?}

Developing new drugs. Governmental financial support for innovative antibacterial drug development. Improving diagnosis. Novel technologies for guiding antibiotic treatment. Increasing awareness. Antibiotic stewardship programs to reduce antibiotic consumption.

\section{How can I get involved?}

WHO, alongside the Food and Agriculture Organization of the United Nations and the World Organization for Animal Health, is developing a range of materials that are available to the public? In the meantime, you can continue to spread the word and start planning now by Organizing events or activities in your workplace, community, school, university. Spreading the word - you can find lots of information on sites. Get active on your social media accounts and get your networks involve. Reaching out to your local government representatives, local leaders or influencers and encouraging them to take action on antibiotic resistance. Leading by example: ensure you only take antibiotics if prescribed by a qualified healthcare professional, practice good hand hygiene, and never share or use leftover antibiotics.

\section{Alert to this crisis, the May 2015 World Health Assembly adopted a global action plan on antimicrobial resistance, which outlines the following objectives:}

The WHO Heightening Global Strategies Awareness of the problem to policy makers, health professionals and general public through effective communication, education and training. It strengthens international Surveillance Programs and establishing international links among these programs. Sponsor Projects/ programs to contain the Spread of AR problem, to reduce the incidence of infection through effective sanitation, hygiene and infection prevention measures such as implementation of infection control methods in hospitals and communities. Developed an Antimicrobial Resistance Information Bank (Info Bank) on surveillances activities and addressed to policymakers and aims to persuade governments to take actions. The Alliance, on the 
worldwide level, for the Prudent/optimize Use of Antibiotics in both human and vet. Medicine. Coordinate/provide international forums for international representatives to build collaboration in data collection and for the exchange of ideas.to develop the economic case for sustainable investment that takes account of the needs of all countries and to increase investment in new medicines, diagnostic tools, vaccines and other interventions. Discovery and Development of New Antibiotics, Guide Research, investment in developing novel diagnostic tools and investment in new vaccines.

\section{Conclusion}

MDR a problem today a crisis tomorrow, Global action should be done to stop or at least to slow the spreader of this phenomenon. Nevertheless, increase investment in new medicines, diagnostic tools, vaccines and other interventions are necessarily needed to overcome the problem.

\section{References}

1. AT Maynard, M Huang, WG Rice, DG Covell (1998) Reactivity of the HIV1 nucleocapsid protein p7 zinc finger domains from the perspective of density-functional theory. Proceedings of the National Academy of Sciences of the United States of America 95(20): 11578-11583.

2. The Get Smart Campaign by the US Centers for Disease Control and Prevention (CDC) Materials and References, Antibiotic Prescribing and Use in Doctor's Offices.

3. The Trans Atlantic Task Force on Antimicrobial Resistance (TATFAR).

4. The Alliance for the Prudent Use of Antibiotics (APUA).

5. The European Antibiotic Awareness Day, Burden of infections with resistant bacteria.

6. The European Surveillance of Antimicrobial Consumption (ESAC) European Centre for Disease Prevention and Control.

7. The Canadian Antimicrobial Resistance Alliance (CARA) 